\title{
An $L$-banded approximation to the inverse of symmetric Toeplitz matrices
}

\author{
Romain Benassi \\ Telecom Bretagne
}

\author{
Antonio Pievatolo* \\ CNR IMATI
}

June 30, 2009

\author{
Rainer Göb \\ Würzburg University
}

\begin{abstract}
We apply the banded matrix inversion theorem given by Kavcic and Moura [1] to symmetric Toeplitz matrices. If the inverse is banded with bandwidth smaller than its size, there is a gain in arithmetic complexity compared to the current methods for Toeplitz matrix inversion. Our algorithm can also be used to find an approximation of the inverse matrix even though it is not exactly banded, but only well localized around its diagonal.

Keywords: Symmetric Toeplitz Matrix, Trench Matrix, Matrix Inversion, Banded Matrix, Correlation Matrix

AMS MSC2010 classification: 15A09, 65F05, 65F50
\end{abstract}

\section{Introduction}

The banded matrix inverse theorem [1] gives a way for inverting a matrix explicitly when its inverse is known to be $L$-banded. For $L \geq 0$, we call a matrix so if the elements outside the band defined by the $L$ th lower diagonal and the $L$ th upper diagonal are zero. We consider the particular case of an $N \times N$ symmetric Toeplitz matrix $\Gamma$ with banded inverse, where the latter is referred to as a Trench matrix: by applying the theorem, we have found a quite simple explicit formula for the inverse, requiring $O\left(L^{3}\right)$ flops, which compares favourably with the $O\left(N^{2}\right)$ flops needed for

\footnotetext{
${ }^{*}$ CNR IMATI, Via Bassini 15, 20133 Milano, Italy. Phone \& fax: +39 02 23699538; email: antonio.pievatolo@mi.imati.cnr.it
} 
inverting a symmetric Toeplitz matrix (see [2], Sec. 4.7, where the original Trench's algorithm [3] is reported), when $L<N^{\frac{2}{3}}$.

In general, one does not know in advance whether the inverse of $\boldsymbol{\Gamma}$ is banded. However, if $\boldsymbol{\Gamma}$ is well localized ${ }^{1}$ around its diagonal, then we may expect its inverse to be well localized around its diagonal as well, as suggested by results on the decay of the off-diagonal elements of inverses of such matrices (see [4], [5] and [6]). Thus, provided we are able to find a value $L$ such that the elements of $\boldsymbol{\Gamma}^{-1}$ in diagonals beyond $L$ are negligible, then we can regard $\Gamma^{-1}$ as an $L$-banded matrix and use the banded matrix inverse theorem to find an approximation of it. To this end we provide a simple iterative algorithm that, with little arithmetic complexity, indicates whether the theorem applies (up to a given accuracy) and provides the related value of $L$. Another very important advantage of this type of approach is that the storage for the inverse matrix is reduced dramatically if $L \ll N$, so that very large matrices can be inverted and stored.

In the next section, we illustrate statistical applications where inversion of a symmetric Toeplitz matrix could be required. In Section 3 we state Kavcic and Moura's banded matrix inverse theorem [1] and in Section 4 we show its use for symmetric Toeplitz matrices with banded inverse. We present a method for searching the bandwidth $L$ of the inverse matrix in Section 5. In Section 6 we show numerical examples with specific symmetric Toeplitz matrices. Sections 7 concludes the paper with some short remarks.

\section{Statistical applications}

\subsection{Applications in time series analysis}

The analysis of weakly stationary time series is a major field of application of Toeplitz matrices in statistics. Let $X_{1}, \ldots, X_{s}$ be a segment of weakly stationary time series $\left(X_{t}\right)$. Then the covariances $\gamma(i-j)=\operatorname{Cov}\left[X_{i}, X_{j}\right]$ depend on $i$ and $j$ only through $i-j$. Hence the covariance matrix $\boldsymbol{\Gamma}_{s}=(\gamma(i-j))_{1 \leq i, j \leq s}$ is a Toeplitz matrix. The inversion of the Toeplitz matrix $\boldsymbol{\Gamma}_{s}$ is of interest in three contexts: 1) forecasting, 2) parameter estimation in $\operatorname{AR}(p)$ processes, 3) calculating Gaussian likelihood functions.

\footnotetext{
"Well localized" means that the entries of the matrix have a polynomial or exponential decay as we move away from the diagonal
} 


\subsubsection{Linear predictors for stationary time series}

Let $\phi_{h}=\left(\phi_{1, h}, \ldots, \phi_{s, h}\right)^{\top}$ be the vector of coefficients $\phi_{1, h}, \ldots, \phi_{s, h}$ of the best linear $h$ step ahead predictor $X_{s+h \mid s}=\phi_{1, h} X_{s}+\ldots+\phi_{s, h} X_{1}$ based on the observations $X_{1}, \ldots, X_{s}$. Then $\phi_{h}$ satisfies the system $\Gamma_{s} \phi_{h}=\gamma_{h}$ of Yule-Walker type, see [14], where $\gamma_{h}=(\gamma(h), \ldots, \gamma(s+h-1))^{\top}$.

Various algorithms have been suggested for the solution of Yule-Walker equations, for instance, the algorithm by Durbin [7] and Levinson [8], the Berlekamp [9] and Massey [10] algorithm originating in coding theory, and several algorithms suggested by [11]. All these methods belong to the same overall complexity class $O\left(s^{2}\right)$, but they differ considerably in their particular performance, see [12], [11], and [13]. Alternatively, the Yule-Walker equations may also be solved by inverting the Toeplitz matrix $\boldsymbol{\Gamma}_{s}$, e. g., by Trench's algorithm, and letting $\boldsymbol{\phi}_{h}=\boldsymbol{\Gamma}_{s}^{-1} \boldsymbol{\gamma}_{h}$. Trench's algorithm and later refinements thereof also belong to the $O\left(s^{2}\right)$ class.

In a situation where the inverse $\boldsymbol{\Gamma}_{s}^{-1}$ can be approximated sufficiently well by an $L$ banded matrix with $L<s^{\frac{2}{3}}$, the inversion of the Toeplitz matrix $\boldsymbol{\Gamma}_{s}$ by an algorithm with complexity $O\left(L^{3}\right)$ is a competitive alternative to the customary methods of solving the Yule-Walker equations.

\subsubsection{Estimating autoregressive coefficients in $\operatorname{AR}(p)$ processes}

Consider the problem of estimating the vector $\phi=\left(\phi_{1}, \ldots, \phi_{p}\right)^{\top}$ of coefficients of an autoregressive process $\left(X_{t}\right)$ defined by the recursion $X_{t}=\phi_{1} X_{t-1}+\ldots+\phi_{p} X_{t-p}+\varepsilon_{t}$ from a sample of observations $X_{1}, \ldots, X_{n}$. Let $\widehat{\boldsymbol{\Gamma}}_{p}=(\widehat{\gamma}(i-j))_{1 \leq i, j \leq p}$ be the sample covariance matrix, and let $\widehat{\gamma}_{p}=(\widehat{\gamma}(0), \ldots, \widehat{\gamma}(p))^{\top}$ be the vector of the sample covariances at lags $0, \ldots, p$. Then the so-called Yule-Walker estimator $\widehat{\phi}$ is defined as the solution of the equation $\widehat{\boldsymbol{\Gamma}}_{p} \widehat{\boldsymbol{\phi}}=\widehat{\gamma}$, see [14]. The methods for the solution of this problem are the same as discussed in paragraph 2.1.1, above. Directly inverting $\widehat{\boldsymbol{\Gamma}}_{p}$ by an algorithm of complexity $O\left(L^{3}\right)$ is competitive, if the band limit $L$ of a good approximation of $\widehat{\boldsymbol{\Gamma}}_{p}^{-1}$ is known to be smaller than $p^{\frac{2}{3}}$.

\subsubsection{Calculating Gaussian likelihood functions}

Let $X_{1}, \ldots, X_{n}$ be a segment from a zero mean stationary Gaussian process $\left(Y_{t}\right)$ with covariance function $\gamma(|s-t|)=\operatorname{Cov}\left[Y_{s}, Y_{t}\right]$. The covariance matrix $\boldsymbol{\Gamma}=(\gamma(\mid s-$ $t \mid))_{1 \leq s, t \leq n}$ of the vector $\mathbf{X}=\left(X_{1}, \ldots, X_{n}\right)^{\top}$ is a Toeplitz matrix. The joint density or likelihood of $\mathbf{X}$ as a function of $\boldsymbol{\Gamma}$ is

$$
L_{\boldsymbol{X}}(\boldsymbol{\Gamma})=(2 \pi)^{n / 2}(\operatorname{det} \boldsymbol{\Gamma})^{-1 / 2} \exp \left(-0.5 \mathbf{X}^{\top} \boldsymbol{\Gamma}^{-1} \mathbf{X}\right) .
$$


To avoid the inversion of $\boldsymbol{\Gamma}$ it is often recommended to calculate the likelihood function recursively by the innovations algorithm, see [14]. However, the innovations algorithm is of complexity $O\left(n^{3}\right)$, see [13]. In cases where the inverse $\boldsymbol{\Gamma}$ can be at least approximated by an $L$-banded matrix with $L<n$, it is better to invert $\boldsymbol{\Gamma}$ with a method of complexity $O\left(L^{3}\right)$.

\section{$2.2 \quad$ Weighted least squares}

Linear models are widely applied in statistical data analysis. Consider an observation $Y$ which is assumed to depend on the values $x_{1}, \ldots, x_{p}$ of $p$ identified factors and, additionally, of unspecified random influences which are expressed by a residual $\varepsilon$ which is zero on the average, i. e., $E[\varepsilon]=0$. A linear model relates these quantities by the model equation $Y=\mathbf{x}^{\top} \boldsymbol{\epsilon}+\varepsilon$ where $\mathbf{x}=\left(x_{1}, \ldots, x_{p}\right)^{\top}$ is the vector of factor values and where $\boldsymbol{\epsilon}=\left(\beta_{1}, \ldots, \beta_{p}\right)^{\top}$ is the associated vector of linear coefficients. Consider a sample of observations $Y_{1}, \ldots, Y_{n}$ where $Y_{i}$ follows the linear model equation $Y_{i}=\mathbf{x}_{i}^{\top} \boldsymbol{\epsilon}+\varepsilon_{i}$. In matrix notation, the sample model is $\mathbf{Y}=\mathbf{X} \boldsymbol{\epsilon}+\boldsymbol{\beta}$ where $\mathbf{Y}=$ $\left(Y_{1}, \ldots, Y_{n}\right)^{\top}, \mathbf{X}=\left(\mathbf{x}_{1}, \ldots, \mathbf{x}_{n}\right)^{\top}$, and $\boldsymbol{\beta}=\left(\varepsilon_{1}, \ldots, \varepsilon_{n}\right)^{\top}$.

In practice, the factor values are known whereas the coefficient vector $\boldsymbol{\epsilon}$ is unknown and has to be estimated from the sample data. For this purpose, the simple ordinary least squares $(O L S)$ approach assumes the $Y_{i}$ to be uncorrelated and homogeneous in variance, and determines an estimator $\widehat{\boldsymbol{\epsilon}}$ by minimising with respect to $\boldsymbol{\epsilon}$ the squared distance $(\mathbf{Y}-\mathbf{X} \boldsymbol{\epsilon})^{\top}(\mathbf{Y}-\mathbf{X} \boldsymbol{\epsilon})$ between the observation vector and its mean $\mathbf{X} \epsilon$. However, data often exhibit association and inhomogeneous dispersion. To account for such phenomena, the weighted least squares (WLS) approach determines the estimator $\widehat{\boldsymbol{\epsilon}}$ by minimising, with respect to $\boldsymbol{\epsilon}$, the squared distance $(\mathbf{Y}-\mathbf{X} \boldsymbol{\epsilon})^{\top} \boldsymbol{\Gamma}^{-1}(\mathbf{Y}-\mathbf{X} \boldsymbol{\epsilon})$ weighted by the inverse of the covariance matrix $\boldsymbol{\Gamma}=\operatorname{Cov}[\boldsymbol{\beta}]=\operatorname{Cov}[\mathbf{Y}]$. Provided that the matrix $\mathbf{X}$ is of full rank, the resulting estimator is $\widehat{\boldsymbol{\epsilon}}=\left(\mathbf{X}^{\top} \boldsymbol{\Gamma}^{-1} \mathbf{X}\right)^{-1} \mathbf{X}^{\top} \boldsymbol{\Gamma}^{-1} \mathbf{Y}$, see [15].

Particularly for time indexed data $Y_{1}, \ldots, Y_{n}$, serial association is the more relevant problem than variance inhomogeneity. In case of variance homogeneity, the covariance matrix is a Toeplitz matrix, and the determination of the estimator $\widehat{\boldsymbol{\epsilon}}=\left(\mathbf{X}^{\top} \boldsymbol{\Gamma}^{-1} \mathbf{X}\right)^{-1} \mathbf{X}^{\top} \boldsymbol{\Gamma}^{-1} \mathbf{Y}$ requires a method for the inversion of Toeplitz matrices.

\subsection{Graduation by moving averages}

In actuarial science, smoothing of time indexed data $X_{t}$ is denoted by the term graduation. Consider a series $X_{1}, \ldots, X_{N}$ of time indexed data. The smoothed outputs 
$Y_{1}, \ldots, Y_{N}$ of a centered moving average of regular length $q$ are defined by

$$
Y_{t}=\sum_{\substack{-q \leq i \leq q \\ 1 \leq t-i \leq N}} \alpha_{t, i} X_{t-i}
$$

with weights $\alpha_{t, i}$ summing up to 1 . In the interior $X_{q+1}, \ldots, X_{N-q}$ of the data set, we have $2 q+1$ weights which are symmetrical and invariant in $t$, i. e., $\alpha_{t,-i}=\alpha_{-i}=\alpha_{i}=$ $\alpha_{t, i}$ for $t=q+1, \ldots, N-q$. A classical and widely used instance is [16] symmetrical 15 -term average

$$
\begin{aligned}
Y_{t}= & \frac{1}{320}\left(-3 X_{t-7}-6 X_{t-6}-5 X_{t-5}+3 X_{t-4}+21 X_{t-3}+46 X_{t-2}+67 X_{t-1}+\right. \\
& \left.+74 X_{t}+67 X_{t+1}+46 X_{t+2}+21 X_{t+3}+3 X_{t+4}-5 X_{t+5}-6 X_{t+6}-3 X_{t+7}\right)
\end{aligned}
$$

At the two extremities $X_{1}, \ldots, X_{q}$ and $X_{N-q+1}, \ldots, X_{N}$ of the data set, there are less than $2 q+1$ weights, and weights cannot be symmetrical. The investigation of strategies for smoothing, with particular attention for the extremities, has lead to analysing graduation from a matrix point of view, see [17]. Let $\mathbf{X}=\left(X_{1}, \ldots, X_{N}\right)^{\top}$ be the vector of observations, let $\mathbf{Y}=\left(Y_{1}, \ldots, Y_{N}\right)^{\top}$ be the vector of smoothed values. The $N \times N$ graduation matrix $\mathbf{G}$ is constructed from the weights of the moving average in the following way: The $t$-th row of $\mathbf{G}$ starts with $t-q-1$ leading zeroes, followed by the weights $\alpha_{t, \max \{t-q, 1\}}, \ldots, \alpha_{t, \min \{t+q, N\}}$, followed by zeroes to complete $N$ positions. Then we have $\mathbf{Y}=\mathbf{G X}$. Greville [17] shows that, under several regularity conditions on the properties of the moving average at the extremities of the data, the graduation matrix $\mathbf{G}$ is of the form $\mathbf{G}=\mathbf{I}-\mathbf{K}^{\top} \mathbf{D K}$ where the $(N-s) \times N$-matrix $\mathbf{K}$ is a so-called differencing matrix with the property

$$
\mathbf{K}^{\top} \mathbf{K}=\left((-1)^{j-i}\left(\begin{array}{c}
2 s \\
s+j-i
\end{array}\right)\right)_{1 \leq i, j \leq s}
$$

and where the $(N-s) \times(N-s)$-matrix $\mathbf{D}$ is a Trench matrix. So the problem of finding regular moving averages is related to inverting Toeplitz matrices with strictly banded inverses.

\section{The banded matrix inverse theorem}

This part introduces some notation and re-states the result obtained by [1]. If we consider an $N \times N$ matrix $\mathbf{A}$, a principal submatrix of $\mathbf{A}$, spanning columns and rows $i$ through $j(1 \leq i \leq j \leq N)$ is denoted by $\mathbf{A}_{j}^{i}$ (in MatLab notation: $\mathbf{A}(i: j, i: j)$ ). 
In the following, we need to consider some overlaps, especially for submatrix blocks. The following example, taken from [1], illustrates the overlap algebra via a $3 \times 3$ example of a tridiagonal matrix $(L=1)$ :

$$
\left.\left[\begin{array}{ccc}
a_{1} & a_{2} & 0 \\
a_{3} & x+y+z & a_{4} \\
0 & a_{5} & a_{6}
\end{array}\right]=\left[\begin{array}{ll}
a_{1} & a_{2} \\
a_{3} & x
\end{array}\right]+y+\begin{array}{cc}
z & a_{4} \\
a_{5} & a_{6}
\end{array}\right]
$$

Definition ( $L$-Band Extension): The matrix $\mathbf{R}$ is called the $L$-band extension of an $N \times N$ matrix $\mathbf{C}$, if its inverse $\mathbf{R}^{-1}$ is related to $\mathbf{C}$ as shown below.

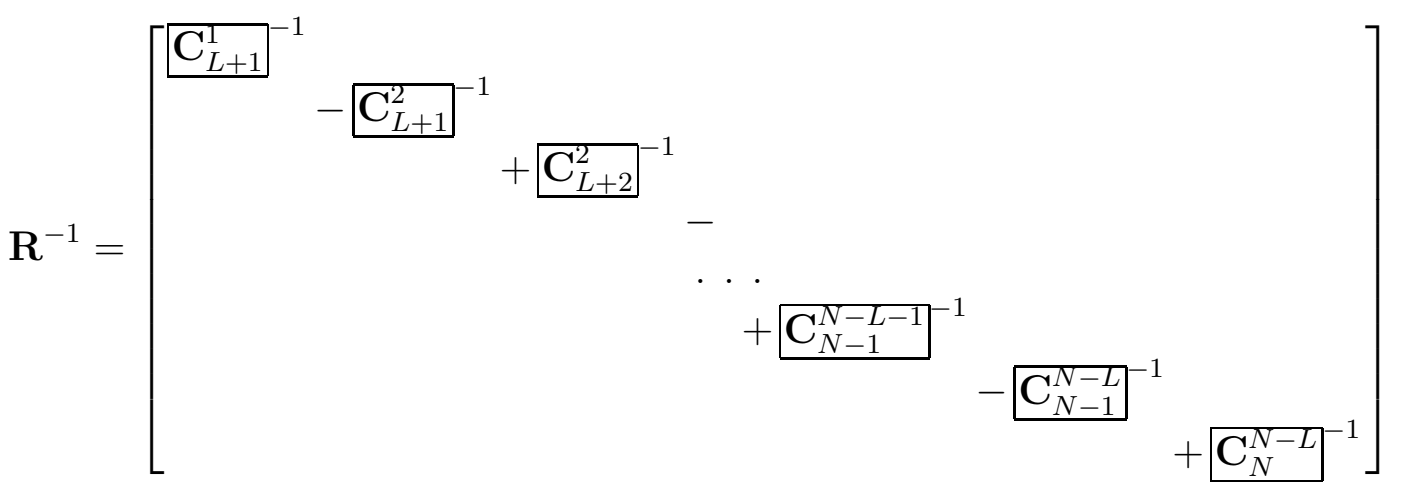

Theorem 1 (banded matrix inverse theorem): $\mathbf{R}^{-1}$ is $L$-banded if and only if $\mathbf{R}$ equals its own $L$-band extension.

The proof is given in [1].

\section{Inverting a symmetric Toeplitz matrix when the inverse is $L$-banded}

We apply the banded matrix inverse theorem to calculate the inverse of a symmetric Toeplitz matrix when its inverse is known to be $L$-banded.

Let $\boldsymbol{\Gamma}$ be an $N \times N$ symmetric Toeplitz matrix with $L$-banded inverse $\boldsymbol{\Gamma}^{-1}$. From the banded matrix inverse theorem, we know that $\Gamma$ equals its own $L$-band extension, 
that is,

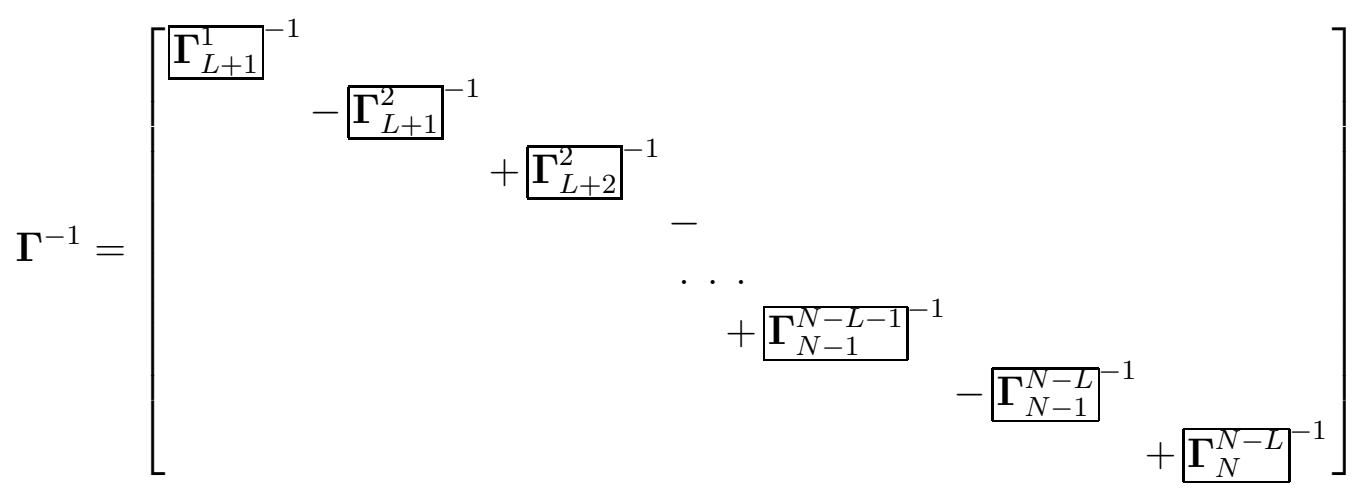

Any block is added (or subtracted) in the position given by the principal submatrix identified by the subscript and the superscript that identifies the block. Since $\boldsymbol{\Gamma}$ is a Toeplitz matrix, then for all $i$ where $1 \leq i \leq N-L$ :

$$
\Gamma_{L+1}^{1}=\Gamma_{L+i}^{i}
$$

and for $1 \leq i<N-L$

$$
\Gamma_{L+1}^{2}=\Gamma_{L+i}^{i+1} \text {. }
$$

As we can see in the expression of $\boldsymbol{\Gamma}^{-1}$, it is always the same pattern that appears. In the second part of this section we will explain how the determination of $\left(\boldsymbol{\Gamma}^{-1}\right)_{L+1}^{1}$ is sufficient for finding all the coefficients of $\boldsymbol{\Gamma}^{-1}$, provided that $L+1<N-L$, so we address this problem first.

To find $\left(\boldsymbol{\Gamma}^{-1}\right)_{L+1}^{1}$, the only thing to do is to use the expression given for $\boldsymbol{\Gamma}^{-1}$ and to mind the overlap. In the pictures below the coefficients do not appear. These pictures are here mainly to help understand the overlap pattern.

At the "beginning" (before taking the overlaps into account) we only have $\left[\boldsymbol{\Gamma}_{L+1}^{1}\right]^{-1}$ :

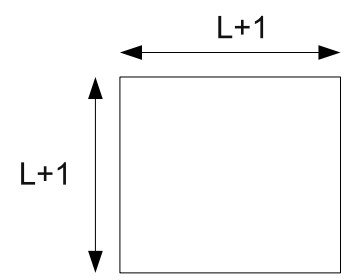

Then we have to add $-\left[\boldsymbol{\Gamma}_{L+1}^{2}\right]^{-1}$ using the overlap definition: 


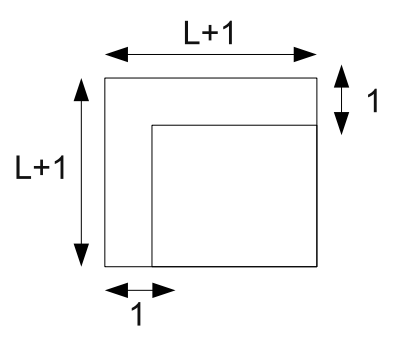

$\left[\boldsymbol{\Gamma}_{L+1}^{1}\right]^{-1}$ is now added in the overlapping way:

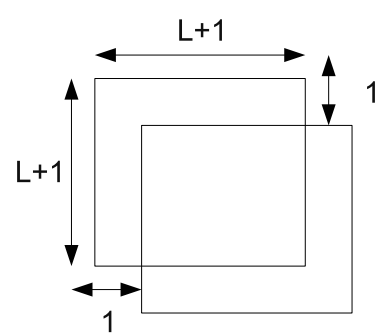

We follow once again the same pattern, with the addition of $-\left[\boldsymbol{\Gamma}_{L+1}^{2}\right]^{-1} \ldots$

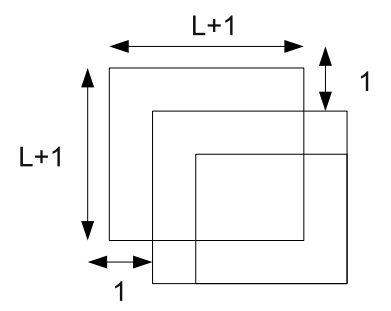

$\ldots$ and the addition of $\left[\Gamma_{L+1}^{1}\right]^{-1}$

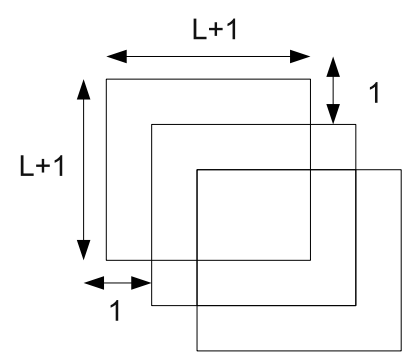

This pattern is applied $L$-times ( $L$ additions of $\left[\boldsymbol{\Gamma}_{L+1}^{1}\right]^{-1}$ and $L$ additions of $-\left[\boldsymbol{\Gamma}_{L+1}^{2}\right]^{-1}$ ): 


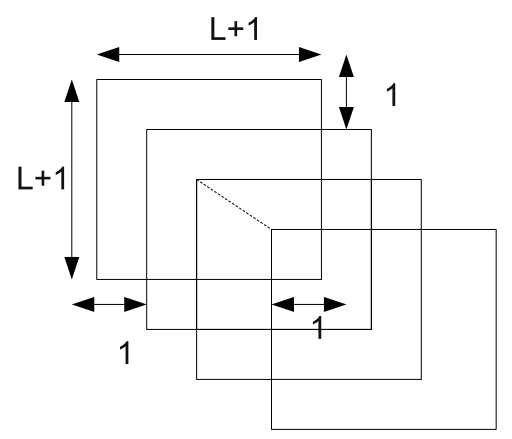

Now, all the coefficients of $\left(\boldsymbol{\Gamma}^{-1}\right)_{L+1}^{1}$ are known, so all the coefficients of $\left(\boldsymbol{\Gamma}^{-1}\right)_{N}^{N-L}$ are also known. Indeed, $\boldsymbol{\Gamma}$ is a symmetric Toeplitz matrix and so is doubly symmetric (that is to say symmetric about both the main diagonal and the secondary diagonal), so its inverse is also doubly symmetric (see [2]). It is now simple to see, in looking at the expression of $\boldsymbol{\Gamma}^{-1}$, that for $k$ and $i$ with $1 \leq k \leq L+1$ and $k \leq i \leq L+1$ we have:

$$
\left[\left(\boldsymbol{\Gamma}^{-1}\right)_{N}^{N-L}\right]_{i, k}=\left[\left(\boldsymbol{\Gamma}^{-1}\right)_{L+1}^{1}\right]_{L+2-i, L+2-k} .
$$

So the upper half of $\left(\boldsymbol{\Gamma}^{-1}\right)_{N}^{N-L}$ is complete and the other coefficients are found thanks to the symmetry property. Now the elements represented in the following picture have been filled.

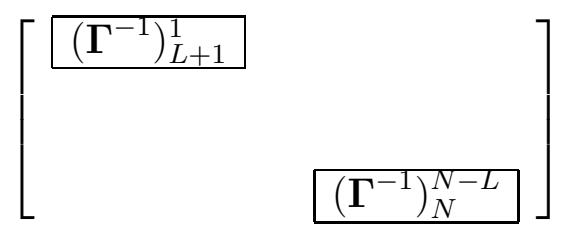

It remains to determine the coefficients situated between this two blocks. To this end, we introduce the following notation:

$$
\begin{gathered}
\boldsymbol{\Gamma}=\left[\gamma_{i, j}\right]_{1 \leq i, j \leq N} \\
\boldsymbol{\Gamma}^{-1}=\left[\gamma_{i, j}^{-1}\right]_{1 \leq i, j \leq N}
\end{gathered}
$$

(Notice that here $\gamma_{i, j}^{-1} \neq \frac{1}{\gamma_{i, j}}$ )

$$
\begin{gathered}
\Gamma_{L+1}^{1}{ }^{-1}=\left[a_{i, j}\right]_{1 \leq i, j \leq L+1} \\
\Gamma_{L+1}^{2}{ }^{-1}=\left[b_{i, j}\right]_{1 \leq i, j \leq L}
\end{gathered}
$$




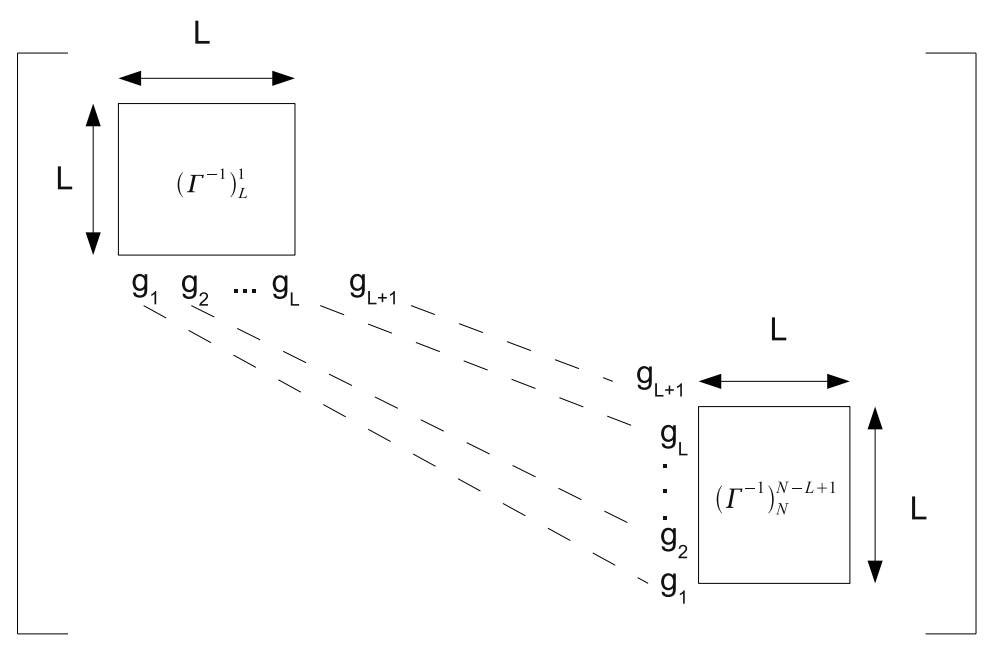

Figure 1: The structure of $\boldsymbol{\Gamma}^{-1}$

We can notice that the $L$-band of $\boldsymbol{\Gamma}^{-1}$, excluding the entries of $\left(\boldsymbol{\Gamma}^{-1}\right)_{L+1}^{1}$ and $\left(\boldsymbol{\Gamma}^{-1}\right)_{N-L}^{L}$, has a Toeplitz structure (that is, each descending diagonal from left to right is constant). For example, thanks to the previous pictures of the overlapping patterns and the expression of $\Gamma^{-1}$, it is possible to see that for $L+1 \leq i \leq N-L$, we have on the diagonal:

$$
\begin{aligned}
& \gamma_{i, i}^{-1}=\gamma_{L+1, L+1}^{-1}=a_{L+1, L+1}-b_{L, L}+a_{L, L}-b_{L-1, L-1}+\ldots-b_{1,1}+a_{1,1} \\
&= \\
& \sum_{j=1}^{L+1} a_{j, j}-\sum_{j=1}^{L} b_{j, j} .
\end{aligned}
$$

This happens because $\gamma_{L+1, L+1}^{-1}$ is the result of a complete overlap pattern, which then repeats itself identically until there is room in the matrix for it.

The same reasoning can be applied to the entries of the last row of $\left(\boldsymbol{\Gamma}^{-1}\right)_{L+1}^{1}$, so that, proceeding diagonal-wise on the sub-diagonals, we have, for any $k=1, \ldots, L$,

$$
\gamma_{i, i+k-(L+1)}^{-1}=\gamma_{L+1, k}^{-1}=\sum_{j=0}^{k-1} a_{L+1-j, k-j}-\sum_{j=0}^{k-2} b_{L-j, k-j-1}, \quad L+2 \leq i \leq N-k .
$$

This expression gives the values of the subdiagonals only, but the matrix is symmetric, so we can represent the complete matrix in Figure 1, where $g_{i}=\gamma_{L+1, i}^{-1}$. This algorithm will be called Algorithm $(\boldsymbol{\Gamma}, L)$ in the following. As far as the flop count is concerned, the algorithm requires the inversion of $\Gamma_{L+1}^{1}$ and $\Gamma_{L+1}^{2}$, which are both 
symmetric Toeplitz matrices, so that $O\left(L^{2}\right)$ flops are required. The overlap additions, are done with $O\left(L^{3}\right)$ flops, even exploiting the symmetry of the blocks. The first addition of $\left(\boldsymbol{\Gamma}_{L+1}^{2}\right)^{-1}$ takes in fact $L(L+1) / 2$ flops, then the addition of the appropriate submatrix of $\left(\boldsymbol{\Gamma}_{L+1}^{1}\right)^{-1}$ takes $(L-1) L / 2$ flops and so on. Proceeding in this way we accumulate $O\left(L^{3}\right)$ additions.

In the case $L+1 \geq N-L$, the inverse matrix can still be recovered from $\left(\boldsymbol{\Gamma}^{-1}\right)_{L+1}^{1}$, but the subdiagonals can be filled using the elements of its last row only for the entries above and including the secondary diagonal. The remaining entries can be filled by using the double symmetry property.

\section{The bandwidth size for the approximation of the inverse matrix}

In this section, we try to find a way to determine a bandwidth value $L$ such that the application of the matrix inversion theorem provides a good approximation to the true inverse $\boldsymbol{\Gamma}^{-1}$. For $\boldsymbol{\Gamma}$, we consider symmetric Toeplitz matrices that are well localized around their diagonals. This particular choice is motivated by applications in statistics, where covariance or correlation matrices are in fact (positive definite and) symmetric; moreover, if we can write $\gamma_{i j}=\gamma(|i-j|)$, where $\gamma(\cdot)$ can be for example the autocorrelation function of a stationary stochastic process, then $\Gamma$ is also Toeplitz. The matrix is also well localized around its diagonal if $|\gamma(k)|$ decays at a polynomial or exponential rate. Since we are dealing with finite matrices, we focus on the case where the entries become negligible for $k>p$, with $p$ much smaller than $N$, the size of the matrix.

Some relations between the decay rate of the entries of a matrix while moving away from the diagonal and the corresponding rate of its inverse exist. One of the most famous results (given in [6]) concerns matrices with exponential or polynomial decay. Let $\mathbf{A}=\left[\mathbf{A}_{k, l}\right]_{k, l \in \mathcal{I}}$, where $\mathcal{I}=\mathbb{Z}, \mathbb{N}$ or $\{0, \ldots, N-1\}$ :

(i) A belongs to $\mathcal{E}_{\gamma}$ if, for $\gamma>0$ and $c>0$,

$$
\left|\mathbf{A}_{i, j}\right| \leq c e^{-\gamma|i-j|}
$$

(ii) A belongs to $\mathcal{Q}_{s}$ if, for $s>1$ and $c>0$,

$$
\left|\mathbf{A}_{i, j}\right| \leq c(1+|i-j|)^{-s} .
$$

Let $\mathbf{A}: l^{2}(\mathcal{I}) \rightarrow l^{2}(\mathcal{I})$ be an invertible matrix where $\mathcal{I}=\mathbb{Z}, \mathbb{N}$ or $\{0, \ldots, N-1\}$, then the following results hold: 
(a) if $\mathbf{A} \in \mathcal{E}_{\gamma}$, then for some $\gamma_{1}<\gamma$

$$
\mathbf{A}^{-1} \in \mathcal{E}_{\gamma_{1}}
$$

(b) if $\mathbf{A} \in \mathcal{Q}_{s}$, then

$$
\mathbf{A}^{-1} \in \mathcal{Q}_{s}
$$

Even though these results may be very useful when the matrices considered are infinite, they are useless when the matrices are finite. In principle, provided $\gamma_{1}, c$ and $s$ are given, one can find a value of $|i-j|$ such that $\left|\mathbf{A}_{i j}^{-1}\right|$ is smaller than a given threshold, and consequently a sensible candidate value for $L$. However it is difficult to find values that give a tight bound and, in particular, the result is trivially true for finite matrices when $c$ is large enough.

Another famous relation between the decay rate of a matrix and of its inverse is given in [4]. Let $\mathbf{A}$ hermitian positive definite and s-banded with:

$$
K=\|\mathbf{A}\| \cdot\left\|\mathbf{A}^{-1}\right\|, \quad q=\frac{\sqrt{K}-1}{\sqrt{K}+1} \quad \text { and } \quad \lambda=q^{\frac{1}{s}}
$$

then:

$$
\left|\mathbf{A}_{k, l}^{-1}\right| \leq c \lambda^{|k-l|}
$$

where $c=\left\|\mathbf{A}^{-1}\right\| \max \left\{1, \frac{(1+\sqrt{K})^{2}}{2 K}\right\}$ and $\|$.$\| is the 2-norm. Again the bound is$ not tight enough, in that a simple numerical experiment with a banded symmetric Toeplitz matrix showed that it provides values of $L$ that are much larger than necessary. Moreover, the determination of $c$ requires the computation of $\left\|\mathbf{A}^{-1}\right\|$, which is demanding.

Other approximations are given in [5] when the matrix is banded and "near to Toeplitz", but again it cannot be useful because when the matrix considered is symmetric (this is our case) these approximations are not better than those in [4].

Another possibility is to consider an arbitrary value of $L$, to compute an inverse $\widehat{\Gamma^{-1}}$ with the method of the previous section, and to check whether it approximates $\Gamma^{-1}$ well enough. If it does not, another larger value of $L$ can be considered and so on. Thanks to the very particular structure of $\widehat{\Gamma^{-1}}$, it is possible to test whether it is a good approximation with little computation, so that the testing can be done repeatedly with different values of $L$.

Let $\boldsymbol{\Gamma}$ be a covariance matrix of size $N$ and with negligible (or zero) entries outside a band of width $p$ and let $r$ be an upper bound for

$$
\max _{i j}\left|\left[\widehat{\Gamma} \widehat{\boldsymbol{\Gamma}^{-1}}\right]_{i j}-\delta_{i j}\right|
$$


where $\delta_{i j}=1$ if $i=j$ and zero otherwise. Let $\mathbf{e}_{j}^{N}$ the $N \times 1$ vector with $\mathbf{e}_{j}^{N}(i)=\delta_{i j}$. A trivial but computationally demanding algorithm for finding a value of $L$ is:

Algorithm 1: Input $(\boldsymbol{\Gamma}, L)$

Let $\widehat{\boldsymbol{\Gamma}_{L}^{-1}}=\operatorname{Algorithm}(\boldsymbol{\Gamma}, L)$ and $\widehat{\boldsymbol{\Gamma}_{L}^{-1}}(j)$ its jth column.

If

$$
\forall j \quad \text { with } \quad 1 \leq j \leq N, \quad \max _{j} \max _{i}\left|\left[\boldsymbol{\Gamma} \widehat{\boldsymbol{\Gamma}_{L}^{-1}}\right]_{i j}-\mathbf{e}_{j}^{N}(i)\right| \leq r
$$

then

$$
\operatorname{return} \widehat{\Gamma_{L}^{-1}}
$$

else

$$
L=L+\text { increment }
$$

end.

$\operatorname{Algorithm}(\boldsymbol{\Gamma}, L)$ is defined at the end of the previous section. For the initialization step of the algorithm, we can consider $L=p$. The bandwidth can be increased up to $L=N-1$, that is, the banded matrix inversion theorem applies trivially by inverting the full matrix in one step.

This algorithm is based on the fact that the multiplication of a matrix by its inverse gives the identity matrix. We can avoid the computation of (1) for all values of $j$ to obtain a nearly similar algorithm at a far lower cost. Indeed, as seen before, between columns $L+1$ and $N-L, \widehat{\Gamma_{L}^{-1}}$ has a kind of Toeplitz structure (see Figure 1) and, as $\boldsymbol{\Gamma}$ is a Toeplitz matrix, we find that $\max _{i}\left|\left[\boldsymbol{\Gamma} \widehat{\boldsymbol{\Gamma}_{L}^{-1}}\right]_{i j}-\mathbf{e}_{j}^{N}(i)\right|$ has the same value for $L+1 \leq j \leq N-L$ so we can consider $j=L+1$. If the true $\Gamma^{-1}$ really has this structure we expect this check to be sufficient because, by construction, the remaining columns have no more than $2 L+1$ nonzero entries. On the other hand, if the algorithm fails, this means that $\Gamma^{-1}$ has a different type of structure and the banded matrix inverse theorem does not apply.

It is now obvious that we need not compute the whole $\widehat{\Gamma_{L}^{-1}}$, but only its column $L+1$. If we call this column $\mathbf{c}$, we have:

$$
\mathbf{c}=\left[c_{1}, c_{2}, \ldots, c_{L}, c_{L+1}, c_{L}, \ldots, c_{2}, c_{1}, 0, \ldots, 0\right]^{\top}
$$

with, as shown in the previous section (and with the same notation), for $0 \leq k \leq L$ :

$$
c_{L+1-k}=\left[\widehat{\boldsymbol{\Gamma}_{L}^{-1}}\right]_{L+1-k, L+1}=\sum_{j=1}^{L+1-k} a_{j+k, j}-\sum_{j=1}^{L-k} b_{j+k, j} .
$$


The calculation of vector c requires $O\left(L^{2}\right)$ flops.

The new algorithm replacing Algorithm 1 is then as follows:

Algorithm 2: Input $(\boldsymbol{\Gamma}, L)$

Let $\widehat{\boldsymbol{\Gamma}^{-1}}=\operatorname{Algorithm}(\boldsymbol{\Gamma}, L)$ and $c=\widehat{\boldsymbol{\Gamma}^{-1}}(L+1)$ its $(L+1)-$ th column .

If

$$
\max _{i}\left|[\boldsymbol{\Gamma} \mathbf{c}](i)-\mathbf{e}_{L+1}^{N}(i)\right| \leq r
$$

then

$$
\operatorname{return} \widehat{\Gamma_{L}^{-1}}
$$

else

$$
L=L+\text { increment }
$$

end.

For the initialization step of the algorithm, we can consider $L=p$. The value of $L$ can be increased as long as $L+1<N-L$, because for larger values the blocks $\left(\boldsymbol{\Gamma}^{-1}\right)_{L+1}^{1}$ and $\left(\boldsymbol{\Gamma}^{-1}\right)_{N}^{N-L}$ start to overlap and $c$ loses its peculiar structure.

\section{Some results in specific cases}

Here we focus on some specific autocorrelation functions, and apply the algorithm to correlation matrices built from them.

For $|\phi|<1$ let:

$$
f_{\phi}(h)=\phi^{|h|}, \quad h=0, \pm 1, \pm 2, \ldots
$$

and for any real number $\lambda$ :

$$
g_{\lambda}(h)=\frac{3}{32} 2^{-|h|}\left(\frac{32}{3}+\lambda|h|\right), \quad h=0, \pm 1, \pm 2, \ldots,
$$

where

$$
\lambda=\frac{4}{3}(1+2 \theta)+\frac{4}{9}(1+2 \theta)^{2} .
$$

The function $f_{\phi}$ is the autocorrelation function of a stationary and causal autoregressive process of order 1 . The function $g_{\lambda}$ is the autocorrelation of the $\operatorname{ARMA}(2,1)$ stochastic process defined by the equation

$$
X_{t}-X_{t-1}+\frac{1}{4} X_{t-2}=Z_{t}+\theta Z_{t-1}
$$




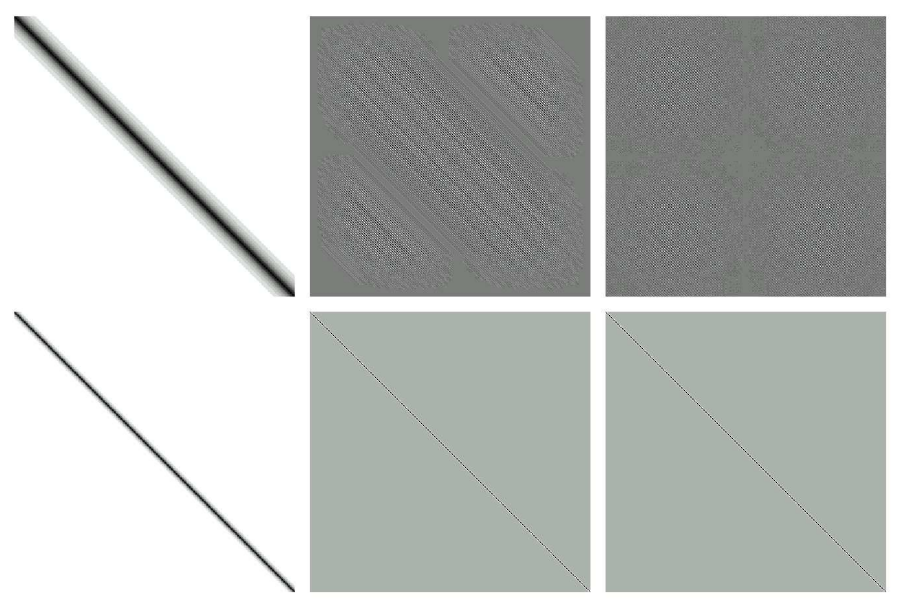

Figure 2: Image plots of correlation matrices derived from $f_{\phi}$ (higher values correspond to darker shades of gray). First row: $\phi=0.9$ and $L=250$. Second row: $\phi=0.7$ and $L=30$. First column: correlation matrix. Second column: banded inverse theorem approximation. Third column: true inverse.

where $Z_{t}$ is a white noise with known variance (see [14], Sec. 3.3). This process is causal; it is also invertible for $|\theta|<1$. In particular, $\lambda=8$ for $\theta=1$ and we will see that the inverse correlation matrix tends to lose its property of being well localized around its diagonal as $\theta$ approaches 1 .

We consider the autocorrelation matrices related to these functions, but beyond a certain lag the correlation is forced to zero. In the following, the size of these matrices is $300 \times 300$ and the lag is 25 so the matrices are banded with bandwidth $p=25$. For a selection of values of $\phi$, the following table indicates the values of $L$ needed to reach a precision of $10^{-6}$, obtained from Algorithm2( $\left.\boldsymbol{\Gamma}, L\right)$, using a starting value $L=p$ and an increment of 5 :

\begin{tabular}{c|ccccc}
$\phi$ & $\leq 0.5$ & 0.7 & 0.8 & 0.85 & 0.9 \\
\hline$L$ & 25 & 30 & 80 & 130 & 299
\end{tabular}

As $\phi$ increases, nonzero elements start to appear away from the diagonal in the inverse matrix and, in particular, with $\phi=0.9$, the inverse matrix is not well localized around the diagonal anymore. On the other hand, if the off-diagonal elements of $\boldsymbol{\Gamma}$ have decayed enough near zero at the border of the $p$-band, then the algorithm is effective (see Figure 2).

A similar type of behaviour occurs with $g_{\lambda}$, for which we consider values near the 


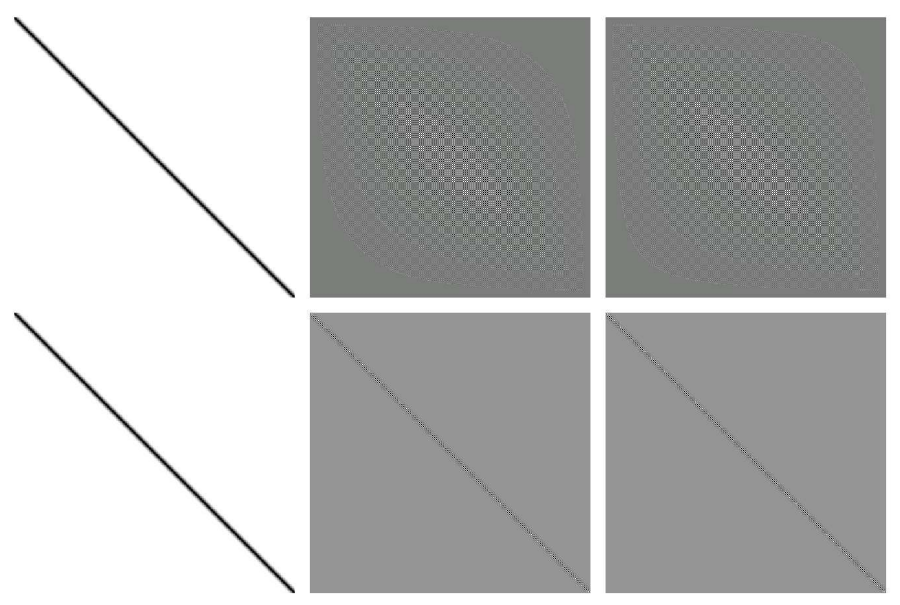

Figure 3: Image plots of correlation matrices derived from $f_{\lambda}$ (higher values correspond to darker shades of gray). First row: $\lambda=8$ and $L=250$. Second row: $\lambda=7.7$ and $L=30$. First column: correlation matrix. Second column: banded inverse theorem approximation. Third column: true inverse.

region of non-invertibility of the $\operatorname{ARMA}(2,1)$ process. The results of Algorithm2( $\boldsymbol{\Gamma}, L)$, with the same values of $p, N$ and $r$ are reported in the following table:

\begin{tabular}{c|ccccc}
$\lambda$ & $\leq 6.2$ & 7.7 & 7.98 & 7.996 & 8 \\
\hline$L$ & 25 & 30 & 80 & 130 & 299
\end{tabular}

and image plots for two values of $\lambda$ appear in Figure 3. The message from this figure is that even though the two correlation matrices are superficially similar, their inverses have a very different structure; also, if the inverse matrix is essentially banded then very few iterations of Algorithm2 $(\boldsymbol{\Gamma}, L)$ are needed.

\section{Conclusion}

We have described an algorithm for inverting an $N \times N$ symmetric Toeplitz matrix $\Gamma$ when its inverse is known to be $L$-banded, which constitutes a very effective application of the banded matrix inverse theorem of [1]. The choice of this particular type of matrix is motivated by numerous statistical applications where the inversion of covariance or correlation matrices can be required and where the solution takes $O\left(N^{2}\right)$ flops. The algorithm is efficient when $L<N^{\frac{2}{3}}$, because the coefficients of $\left(\boldsymbol{\Gamma}^{-1}\right)_{L+1}^{1}$, which can be computed with $O\left(L^{3}\right)$ flops, define $\boldsymbol{\Gamma}^{-1}$ completely. 
The algorithm can be used also when the inverse matrix is essentially banded, that is, the entries outside an $L$-band are negligible. If the value of $L$ is unknown, we have provided a simple iterative algorithm, which converges quickly to an approximation at a given accuracy, provided the inverse matrix is actually essentially banded. In this way it is possible to invert very large matrices using very little storage, because the negligible entries in the inverse matrix are never computed.

With two numerical examples of correlation matrices derived from two specific parameterized autocorrelation functions, we have also shown that there are critical values of the parameter such that the inverse matrix becomes non-banded quickly, with respect to variations in the parameter. This situation is signalled by failure of the algorithm to converge after a few iterations.

\section{Acknowledgments}

This work was set up during a two-week research visit to Würzburg University by the second author, funded by the bilateral agreement between the Consiglio Nazionale delle Ricerche (CNR) and the Deutsche Forschungsgemeinschaft (DFG). The first author's two-month internship at CNR IMATI, from mid-July to mid-September 2008, led to its near-completion.

\section{References}

[1] A. Kavcic and J.M.F Moura, Matrices with Banded Inverses: Inversion Algorithms and Factorization of Gauss-Markov Processes, IEEE Transactions on Information Theory 46 (2000) 1495-1509.

[2] G.H. Golub and C.F. van Loan, Matrix Computations, third ed., The Johns Hopkins University Press, Baltimore and London, 1996.

[3] W.F. Trench, An algorithm for the inversion of finite Toeplitz matrices, Journal of the Society for Industrial and Applied Mathematics 12 (1964) 515-522.

[4] S. Demko, W.F. Moss and P.W. Smith, Decay rates for inverse of band matrices, Math. Comp. 43 (1984) 491-499.

[5] V. Eijkhout and B. Polman, Decay Rate of Banded M-Matrices that are Near Toeplitz Matrices, Linear Algebra and its Applications 109 (1988) 247-277. 
[6] S. Jaffard, Propriétés des matrices "bien localisées" prés de leur diagonale et quelques applications, Annales de l'I.H.P., section C, 7 (1990) 461-476.

[7] J. Durbin, The fitting of time series models, Revue Internationale de Statistique 28 (1960) 233-244.

[8] N. Levinson, (1947) The Wiener (root mean square) error criterion in filter design and prediction, Journal of Mathematical Physics 25 (1947) 261-278.

[9] E.R. Berlekamp, Algebraic Coding Theory, McGraw-Hill, New York, 1968.

[10] J.L. Massey, Shift-register synthesis and BCH decoding, IEEE Transactions of Information Theory, 15 (1969) 122-127.

[11] P. Bondon, Recursive relations for multistep prediction of a stationary time series, Journal of Time Series Analysis 22 (2001) 399-410.

[12] H.M. Zhang and P. Duhamel, On the Methods for Solving Yule-Walker Equations, IEEE Transactions on Signal Processing 40 (1992) 2987-3000.

[13] P. Ranocha, Efficiency of some algorithms for prediction in finite stationary time series, Kybernetika 40 (2004) 703-714.

[14] P.J. Brockwell and R.A. Davis, Time Series: Theory and Methods, second ed., Springer-Verlag, New York, 1991.

[15] R. Christensen, Plane Answers to Complex Questions: The Theory of Linear Models, Springer-Verlag, Berlin, Heidelberg, New York, 1996.

[16] J. Spencer, On the Graduation of the Rates of Sickness and Mortality, Journal of the Institute of Actuaries 38 (1904) 334-343.

[17] T.N.E. Greville, Moving-Weighted-Average Smoothing Extended to the Extremities of the Data. I. Theory, Scandinavian Actuarial Journal, 64 (1981) 39-55. 